\title{
Análise de Estressores para o Paciente em Unidade de Terapia Intensiva*
}

\section{Analysis of Stressors for the Patient in Intensive Care Unit}

\author{
Almir Galvão Vieira Bitencourt', Flávia Branco Cerqueira Serra Neves², Maira Pereira Dantas³, \\ Ligia Carvalho Albuquerque ${ }^{1}$, Rodrigo Morel Vieira de Melo', Alessandro de Moura Almeida', \\ Sydney Agareno ${ }^{4}$, José Mário M. Teles ${ }^{5}$, Augusto M. C. Farias ${ }^{5}$, Otavio H. Messeder
}

\section{RESUMO}

JUSTIFICATIVA E OBJETIVOS: O ambiente hospitalar, especialmente o de uma Unidade de Terapia Intensiva (UTI), devido à complexidade do atendimento prestado, bem como a estrutura física, o barulho, os equipamentos e a movimentação das pessoas, é tido como gerador de estresse para os pacientes. O objetivo deste estudo foi identificar e estratificar os estressores para pacientes internados em UTI, na perspectiva do próprio paciente, familiares e profissionais de saúde.

MÉTODO: Estudo de corte transversal realizado entre junho e novembro de 2004, na UTI geral de hospital privado. A amostra foi composta por três grupos: pacientes (G1), familiares (G2) e um membro da equipe da UTI responsável pelo atendimento

1. Graduandos da Faculdade de Medicina da Bahia (UFBA)

2. Graduando da Escola Bahiana de Medicina e Saúde Pública.

3. Médica intensivista, Plantonista da UTI Geral do Hospital Português, Salvador, BA.

4. Médico intensivista, Coordenador da UTI do Hospital Geral Roberto Santos, Salvador, BA.

5. Médico intensivista, Vice-Coordenador da UTI Geral do Hospital Português, Salvador, BA.

6. Médico intensivista, Coordenador da UTI Geral do Hospital Português, Salvador, BA.

* Recebido da Unidade de Terapia Intensiva do Hospital Geral do Hospital Português, Salvador, BA.

Apresentado em 09 de janeiro de 2007

Aceito para publicação em 05 de fevereiro de 2007

Endereço para correspondência:

Almir Galvão Vieira Bitencourt

Rua Vanderlei Pinho, 181/802, Itaigara

41815-270 Salvador, BA

Fone: (71) 8806-0515 / 3451-4063

E-mail: almirgvb@yahoo.com.br

(C)Associação de Medicina Intensiva Brasileira, 2007 do paciente incluído (G3). Para identificação e estratificação dos fatores estressantes, utilizou-se a Escala de Estressores em UTI (Intensive Care Unit Environmental Stressor Scale - ICUESS). Para cada paciente e participante, foi calculado um escore total de estresse (ETE) pela soma de todas as respostas da escala.

RESULTADOS: Foram incluídos 30 pacientes e participantes em cada grupo. A média de idade foi de: $57,30 \pm 17,61$ anos para o G1; 41,43 $\pm 12,19$ anos para o G2; e 40,82 $\pm 20,20$ anos para o G3. A média do ETS foi: $62,63 \pm 14,01$ para os pacientes; 91,10 $\pm 30,91$ para os familiares; e $99,30 \pm 21,60$ para os profissionais. A média do ETS dos pacientes foi estatisticamente inferior à encontrada nos familiares e nos profissionais de saúde $(p<0,01)$. Os principais estressores para os pacientes foram: ver a família e amigos por apenas alguns minutos do dia; tubos no nariz e/ou boca; e não ter controle de si mesmo.

CONCLUSÕES: A percepção sobre os principais estressores foi diferente entre os três grupos. A identificação destes fatores é importante para a implementação de medidas que possam facilitar a humanização do ambiente da UTI.

Unitermos: cuidados críticos, estresse, humanização da assistência, UTI

\section{SUMMARY}

BACKGROUND AND OBJECTIVES: The hospital environment, especially in Intensive Care Units (ICU), due to the complexity of the assistance, as well as the physical structure, the noise, the equipments and people's movement, is considered as stress generator for the patients. The aim of this study was to identify and stratify the stressful factors for patients at an ICU, in the perspective of the own patient, relatives and health care professionals. 
METHODS: A cross-sectional study was carried out between June and November 2004 in a general ICU of a private hospital. The sample was composed of three groups: patients (G1), relatives (G2) and a member of the ICU health care team responsible for the included patient (G3). In order to identify and stratify the stressful factors, we used the Intensive Care Unit Environmental Stressor Scale (ICUESS). For each individual, a total stress score (TSS) was calculated from the sum of all the answers of the scale.

RESULTS: Thirty individuals were included in each group. The mean age of the three groups was: 57.30 \pm 17.61 years for $\mathrm{G} 1 ; 41.43 \pm 12.19$ for $\mathrm{G} 2$; and 40.82 \pm 20.20 for G3. The mean TSS was $62.63 \pm 14.01$ for the patients; $91.10 \pm 30.91$ for the relatives; and $99.30 \pm 21.60$ for the health care professionals. The patients' mean TSS was statistically lower than mean TSS of relatives and professionals $(p<0.01)$. The most stressful factors for the patients were: seeing family and friends only a few minutes a day; having tubes in their nose and/or mouth; and having no control on oneself.

CONCLUSIONS: The perception of the main stressful factors was different among the three groups. The identification of these factors is important to the implementation of changes that can make the humanization of the ICU environment easier.

Key Words: critical care, humanization of assistance, ICU, stress.

\section{INTRODUÇÃO}

O ambiente hospitalar, especialmente o de Unidade de Terapia Intensiva (UTI), devido à complexidade do atendimento prestado, bem como a estrutura física, o barulho, os equipamentos e a movimentação das pessoas, é tido como gerador de estresse para os pacientes e os familiares ${ }^{1}$. Alterações de ordens psicológica e afetiva, como ansiedade e medo, são freqüentemente encontrados entre os pacientes críticos, tornando relevante a identificação dos estressores que contribuem para o desenvolvimento destes quadros².

A presença de equipamentos estranhos, alarmes, e luminosidade intensa contribuem para o estresse físico e psicológico dos pacientes admitidos em UTI. Os fatores específicos como tubos na boca e/ou nariz, ter dor, comprometimento do sono, não ter controle de si mesmo, limitação de movimentos das mãos ou braços devido aos acessos venosos e não ter explicação sobre o seu tratamento; estão descritos na literatura como os itens mais associados ao desenvolvimento do estresse pelos pacientes ${ }^{3,4}$. Neste contexto, o presente trabalho teve como objetivo identificar e estratificar os estressores para os pacientes internados em UTI, nas suas perspectivas, de seus familiares e da equipe de saúde da UTI, permitindo, através da utilização destas informações, o desenvolvimento de um ambiente mais humanizado.

\section{MÉTODO}

Após a aprovação do Comitê de Ética em Pesquisa do Hospital Português, foi realizado um estudo de corte transversal realizado na UTI Geral durante o período de julho a dezembro de 2004. A população do estudo foi composta pelos pacientes internados na unidade durante esse período, seus familiares e membros da equipe de saúde.

A amostra foi composta por três grupos de entrevistados que preencheram os critérios de inclusão. O grupo 1 (G1) foi composto pelos pacientes internados consecutivamente durante o período estabelecido. O grupo 2 (G2) foi formado por um familiar de cada paciente incluído no estudo. O grupo 3 (G3) foi composto por um membro da equipe da UTI responsável pelo atendimento de cada paciente incluído.

Os critérios de inclusão para os três grupos foram: ter idade superior a 18 anos e consentir em participar do estudo através da assinatura do Termo de Consentimento Livre e Esclarecido. Para ser admitido no estudo, o paciente deveria estar internado por mais de 24 horas na UTI, consciente, alerta e bem-informado, sem nenhum distúrbio neurológico ou psicológico prévio à internação. Os familiares incluídos foram parentes de primeiro grau (pais, irmãos, tios, avós, filhos, primos, ou cônjuges) do paciente entrevistado que tivessem visitado o paciente pelo menos uma vez no curso da internação. Os membros da equipe de saúde eram médicos, enfermeiros, fisioterapeutas ou auxiliares de enfermagem que fossem um dos profissionais responsáveis pelo paciente no mesmo dia da sua entrevista. Foram incluídos somente aqueles pacientes em que um dos familiares (G2) e um dos profissionais responsáveis também aceitassem participar do estudo.

Para identificação e estratificação dos fatores estressantes, foi aplicada a Escala de Estressores em 
Unidades de Terapia Intensiva (Intensive Care Unit Environmental Stressor Scale - ICUESS), validada por Ballard $(1981)^{5}$ e por Narstasy $(1985)^{6}$, traduzida e adaptada culturalmente por Novaes e col. ${ }^{3}$. A escala foi composta por 40 itens escalonados de 1 (não estressante) a 4 (muito estressante). A escala foi aplicada, por estudantes previamente treinados, do $2^{\circ}$ ao $4^{\circ}$ dia de internação, ao paciente, um familiar e um membro da equipe da UTI. Ao familiar e ao membro da equipe solicitou-se preencher a escala de acordo com sua percepção dos fatores estressantes para o paciente. Foi utilizado ainda um questionário contendo informações clínicas e demográficas.

Para tabulação e análise dos dados, foi utilizado o pacote estatístico SPSS (Statistical Package for Social Science) versão 9.0 (SPSS Inc. Chigago, Illinois, USA). Foi realizada análise descritiva para caracterizar a amostra de acordo com as variáveis clínicas e demográficas. Um escore médio foi feito para cada um dos itens da escala a fim de obter um ranking dos fatores mais estressantes. Para cada paciente foi calculado um escore total de estresse (ETE) pela soma de todas as respostas da escala. Os valores foram expressos em média \pm DP, para as variáveis contínuas, e em freqüências absolutas e relativas para as variáveis categóricas. Análise de medidas de dispersão e tendência central e o teste de Kolmogorov-Smirnov foram utilizados para verificar a normalidade de distribuição das variáveis contínuas. Variáveis com distribuição normal eram avaliadas utilizando-se o teste $t$ de Student ou Análise de Variância (ANOVA). As variáveis com outro tipo de distribuição, foram analisadas através de teste de comparação não-paramétricos, utilizando-se os de Mann-Whitney $U$ e Kruskal-Wallis. O nível de significância estatística adotado foi de $5 \%$ $(\alpha=0,05)$.

\section{RESULTADOS}

Foram incluídos 30 indivíduos em cada grupo. A média de idade dos três grupos foi de $57,30 \pm 17,61$ anos (G1); 41,43 $\pm 12,19$ anos (G2); 40,82 $\pm 20,20$ anos (G3). Outras características clínicas e demográficas dos três grupos estão descritas na tabela 1 . A média do ETE foi de $62,63 \pm 14,01$ (G1); 91,10 \pm 30,91 (G2) e $99.30 \pm 21.60$ (G3). A média do ETE do G1 foi significativamente inferior à dos outros dois grupos, não havendo diferença estatística significativa entre estes os G2 e G3 (Figura 1).
Tabela 1 - Características Clínicas e Demográficas dos Pacientes (G1), Familiares (G2) e Profissionais (G3), com o Respectivo Escore Total de Estresse (ETE) Médio para Cada Subgrupo.

\begin{tabular}{|c|c|c|}
\hline Características & $\%$ & ETE médio \\
\hline \multicolumn{3}{|l|}{ G1 - Pacientes } \\
\hline \multicolumn{3}{|l|}{ Sexo } \\
\hline Masculino & 53,3 & 62,88 \\
\hline Feminino & 46,7 & 62,36 \\
\hline \multicolumn{3}{|l|}{ Tipo de tratamento } \\
\hline Clínico & 71,4 & 62,10 \\
\hline Cirúrgico & 28,6 & 66,13 \\
\hline \multicolumn{3}{|l|}{ Internação prévia em UTI } \\
\hline Sim & 50 & 63,33 \\
\hline Não & 50 & 61,93 \\
\hline \multicolumn{3}{|l|}{ Nível educacional } \\
\hline Até $1^{\circ}$ grau completo & 34,5 & 60,00 \\
\hline Pelo menos $2^{\circ}$ grau incompleto & 65,5 & 64,32 \\
\hline \multicolumn{3}{|l|}{ Mortalidade Estimada pelo APACHE II } \\
\hline Inferior a $20 \%$ & 53,6 & 65,93 \\
\hline Superior a $20 \%$ & 46,4 & 58,31 \\
\hline \multicolumn{3}{|l|}{ G2 - Familiares } \\
\hline \multicolumn{3}{|l|}{ Sexo } \\
\hline Masculino & 30 & 91,66 \\
\hline Feminino & 70 & 90,85 \\
\hline \multicolumn{3}{|l|}{ Nível educacional } \\
\hline Até $2^{\circ}$ grau completo & 46,7 & 86,78 \\
\hline Pelo menos $3^{\circ}$ grau incompleto & 53,3 & 94,87 \\
\hline \multicolumn{3}{|l|}{ G3 - Profissionais } \\
\hline \multicolumn{3}{|l|}{ Sexo } \\
\hline Masculino & 13,8 & 97,25 \\
\hline Feminino & 86,2 & 100,80 \\
\hline \multicolumn{3}{|l|}{ Atividade profissional } \\
\hline Médico & 16,7 & 92,40 \\
\hline Enfermeiro & 53,3 & 100,12 \\
\hline Fisioterapeuta & 23,2 & 103,42 \\
\hline Auxiliar de enfermagem & 6,7 & 95,50 \\
\hline
\end{tabular}

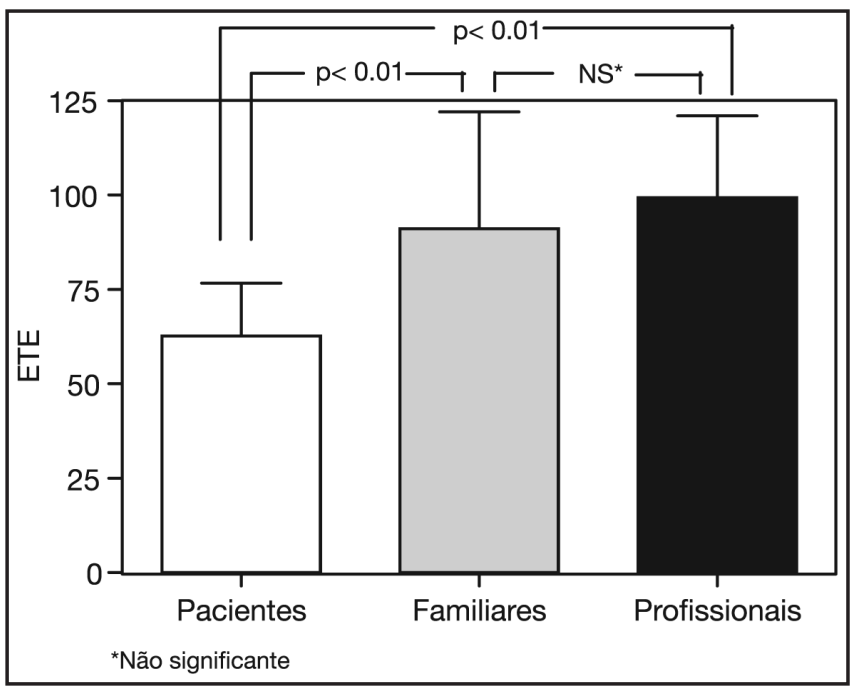

Figura 1 - Média do Escore Total de Estresse (ETE) para Pacientes (G1), Familiares (G2) e Profissionais (G3). 
Não houve diferença estatística significativa no ETE do G1 em relação ao sexo, tipo de tratamento, nível educacional, internação prévia em UTI ou mortalidade estimada pelo APACHE II. Entre os G2 e G3 também não houve diferença estatística significativa no ETE em relação ao sexo, nível educacional para o G1 e atividade profissional para G2.
A tabela 2 apresenta os fatores estressantes com seu respectivo escore médio para cada grupo. Entre os 10 estressores considerados mais importantes para os três grupos, houve uma concordância na maioria deles, no entanto, a ordem destes e o escore médio atribuído a cada um diferiu significativamente. Os três principais fatores estressantes na visão dos pacientes

Tabela 2 - Estressores Avaliados com seu Respectivo Escore Médio para os Pacientes (G1), Familiares (G2) e Profissionais (G3).

\begin{tabular}{|c|c|c|c|}
\hline Estressores & $\begin{array}{c}\text { G1 } \\
\text { Ranking (Média } \pm \text { DP) }\end{array}$ & $\begin{array}{c}\mathrm{G} 2 \\
\text { Ranking (Média } \pm \mathrm{DP})\end{array}$ & $\begin{array}{c}\text { G3 } \\
\text { Ranking (Média } \pm \mathrm{DP})\end{array}$ \\
\hline Ver a família e os amigos por apenas alguns minutos por dia & $1(2.60 \pm 1,30)$ & $4(2,87 \pm 1,22)$ & $8(2,93 \pm 0,94)$ \\
\hline Ter tubos no nariz e/ou na boca & $2(2,43 \pm 1,22)$ & $5(2,77 \pm 0,97)$ & $4(3,18 \pm 0,98)$ \\
\hline Não ter controle de si mesmo & $3(2,27 \pm 1,23)$ & $9(2,69 \pm 1,07)$ & $18(2,66 \pm 1,01)$ \\
\hline Sentir falta do marido ou da esposa & $4(2,20 \pm 1,30)$ & $7(2,76 \pm 1,21)$ & $10(2,86 \pm 0,86)$ \\
\hline Ser picado por agulhas & $5(2,20 \pm 1,19)$ & $27(2,23 \pm 1,07)$ & $3(3,23 \pm 0,94)$ \\
\hline Ter que usar oxigênio & $6(2,17 \pm 1,23)$ & $25(2,23 \pm 1,19)$ & $32(2,13 \pm 0,97)$ \\
\hline Não conseguir dormir & $7(2,03 \pm 1,25)$ & $1(3,10 \pm 1,15)$ & $5(3,14 \pm 0,95)$ \\
\hline Estar preso por tubos & $8(2,03 \pm 1,13)$ & $3(2,97 \pm 1,10)$ & $7(3,00 \pm 1,17)$ \\
\hline Ter dor & $9(2,00 \pm 1,26)$ & $2(3,07 \pm 1,07)$ & $1(3,33 \pm 0,96)$ \\
\hline $\begin{array}{l}\text { Não conseguir mexer as mãos ou os braços devido as vias } \\
\text { venosas }\end{array}$ & $10(2,00 \pm 1,15)$ & $8(2,71 \pm 1,21)$ & $16(2,70 \pm 1,02)$ \\
\hline Ter sede & $11(2,00 \pm 1,20)$ & $10(2,69 \pm 1,07)$ & $15(2,70 \pm 0,95)$ \\
\hline Escutar o barulho e os alarmes dos equipamentos & $12(1,97 \pm 1,03)$ & $15(2,53 \pm 1,01)$ & $2(3,33 \pm 0,96)$ \\
\hline Escutar os alarmes do monitor cardíaco dispararem & $13(1,93 \pm 1,02)$ & $20(2,34 \pm 1,17)$ & $6(3,10 \pm 1,00)$ \\
\hline Ter luz acesa constantemente & $14(1,83 \pm 1,12)$ & $29(2,10 \pm 1,13)$ & $21(2,43 \pm 1,01)$ \\
\hline Ter que ficar olhando para os detalhes do teto & $15(1,73 \pm 1,02)$ & $21(2,31 \pm 1,17)$ & $33(2,07 \pm 0,98)$ \\
\hline Sons e ruídos desconhecidos & $16(1,67 \pm 0,92)$ & $22(2,30 \pm 1,14)$ & $19(2,60 \pm 0,93)$ \\
\hline Não saber onde está & $17(1,69 \pm 1,94)$ & $11(2,69 \pm 1,26)$ & $24(2,37 \pm 1,00)$ \\
\hline Estar num ambiente muito quente ou muito frio & $18(1,50 \pm 1,01)$ & $16(2,52 \pm 0,51)$ & $20(2,60 \pm 0,89)$ \\
\hline Cama e/ou travesseiros desconfortáveis & $19(1,47 \pm 0,90)$ & $13(2,60 \pm 1,22)$ & $14(2,71 \pm 0,81)$ \\
\hline Escutar o gemido de outros pacientes & $20(1,41 \pm 0,87)$ & $6(2,76 \pm 1,21)$ & $12(2,77 \pm 1,04)$ \\
\hline Ser acordado pela enfermagem & $21(1,40 \pm 0,86)$ & $32(1,97 \pm 0,98)$ & $22(2,40 \pm 0,89)$ \\
\hline Não ter privacidade & $22(1,37 \pm 0,89)$ & $17(2,48 \pm 1,24)$ & $11(2,77 \pm 0,97)$ \\
\hline Não saber que dia é hoje & $23(1,37 \pm 0,77)$ & $26(2,23 \pm 1,10)$ & $39(1,80 \pm 0,71)$ \\
\hline Não saber que horas são & $24(1,30 \pm 0,70)$ & $24(2,24 \pm 1,12)$ & $35(1,90 \pm 0,76)$ \\
\hline Não ter explicações sobre o tratamento & $25(1,27 \pm 0,64)$ & $12(2,63 \pm 1,27)$ & $13(2,72 \pm 1,00)$ \\
\hline Ser cuidado por médicos desconhecidos & $26(1,27 \pm 0,58)$ & $35(1,79 \pm 1,11)$ & $29(2,29 \pm 0,90)$ \\
\hline Medir a pressão arterial muitas vezes ao dia & $27(1.27 \pm 0,69)$ & $39(1,57 \pm 0,77)$ & $31(2,17 \pm 0,89)$ \\
\hline Não saber quando as coisas vão ser feitas & $28(1,24 \pm 0,69)$ & $14(2,54 \pm 1,17)$ & $27(2,33 \pm 0,84)$ \\
\hline Ser incomodado & $29(1,20 \pm 0,61)$ & $23(2,24 \pm 1,19)$ & $17(2,66 \pm 0,97$ \\
\hline Enfermagem e médicos falando muito alto & $30(1,20 \pm 0,48)$ & $28(2,14 \pm 1,16)$ & $9(2,90 \pm 0,92)$ \\
\hline $\begin{array}{l}\text { Ter a enfermagem constantemente fazendo tarefas ao redor } \\
\text { do leito }\end{array}$ & $31(1,17 \pm 0,53)$ & $37(1,73 \pm 0,98)$ & $36(1,87 \pm 0,86)$ \\
\hline Ser examinado por médicos e por enfermeiros constantemente & $32(1,17 \pm 0,65)$ & $40(1,52 \pm 0,99)$ & $30(2,24 \pm 0,99)$ \\
\hline Ter máquinas estranhas ao redor & $33(1,17 \pm 0,65)$ & $36(1,73 \pm 1,05)$ & $28(2,30 \pm 0,88)$ \\
\hline Ouvir o telefone tocar & $34(1,13 \pm 0,43)$ & $38(1,60 \pm 0,68)$ & $37(1,83 \pm 0,81)$ \\
\hline $\begin{array}{l}\text { Sentir que a enfermagem está mais atenta aos equipamentos } \\
\text { do que a você }\end{array}$ & $35(1,13 \pm 0,43)$ & $30(2,03 \pm 1,24)$ & $26(2,33 \pm 0,76)$ \\
\hline Ver as bolsas de soro penduradas sobre a cabeça & $36(1,10 \pm 0,40)$ & $33(1,86 \pm 1,06)$ & $38(1,80 \pm 0,81)$ \\
\hline A enfermeira não se apresentar pelo nome & $37(1,10 \pm 0,55)$ & $34(1,83 \pm 1,02)$ & $40(1,80 \pm 0,76)$ \\
\hline Sentir que a enfermagem está muito apressada & $38(1,07 \pm 0,37)$ & $19(2,38 \pm 1,21)$ & $23(2,37 \pm 1,00)$ \\
\hline Ter a equipe falando termos incompreensíveis & $39(1,07 \pm 0,25)$ & $18(2,41 \pm 1,32)$ & $25(2,33 \pm 0,88)$ \\
\hline Sentir odores estranhos & $40(1,03 \pm 0,18)$ & $31(1,97 \pm 1,18)$ & $34(1,97 \pm 0,67)$ \\
\hline
\end{tabular}


foram ver a família e os amigos por apenas alguns minutos por dia, ter tubos no nariz e/ou na boca e não ter controle de si mesmo. Os familiares dos pacientes internados na UTI acreditam que os principais fatores estressantes são: não conseguir dormir, ter dor e estar dependente dos tubos. Para os profissionais da saúde que estavam em contato com estes pacientes, os principais estressores são: ter dor e escutar o barulho e os alarmes dos equipamentos.

\section{DISCUSSÃO}

O estresse é uma situação tensa, fisiológica ou psicológica; que pode afetar as pessoas em todas as suas dimensões. A resposta ao estresse é influenciada pela intensidade, duração e âmbito do agente estressor e pelo seu número presente no momento. Em pacientes internados em UTI, o desenvolvimento de estresse está relacionado a conseqüências psicológicas como ansiedade, depressão, raiva, negação e dependência, além de alterações cognitivas como o desenvolvimento de delirium, anteriormente conhecido como psicose da UTI ${ }^{7}$. Alguns estudos têm sugerido que estas conseqüências neuropsicológicas da internação na UTI podem afetar a qualidade de vida dos pacientes após sua saída da unidade ${ }^{8}$.

Neste estudo, a percepção sobre os principais estressores para o paciente internado na UTI foi diferente entre os três grupos avaliados, sobretudo se comparados os próprios pacientes com os familiares e profissionais. Os familiares e profissionais acreditam que alguns fatores são mais estressantes para o próprio paciente do que ele próprio. Este dado também foi observado por outros autores $3,9,10$ os quais relataram que a avaliação do paciente, quanto aos fatores estressantes em UTI, não coincide com a avaliação dos profissionais que o assistem, sendo que esses tendem a valorizar os estressores com maior intensidade do que os próprios pacientes. A explicação desse fato pode estar na projeção dos sentimentos da equipe e dos familiares do paciente, ao se sensibilizarem com o seu sofrimento'. Em comparação ao estudo de Novaes e colaboradores $^{4}$, que utilizou o mesmo instrumento e critérios de inclusão semelhantes, encontramos escores mais baixos para os diversos estressores nos três grupos avaliados. Este dado pode ser justificado por questões culturais e sociais envolvidas neste tipo de avaliação, visto que as populações avaliadas foram de diferentes regiões.

No sentido de melhorar a qualidade do atendimento prestado aos pacientes internados em UTI, é fundamental que os estressores apontados, principalmente pelos pacientes, sejam discutidos. A questão do contato com familiares já foi estudada em outros trabalhos e alguns autores consideraram que é necessário um plano individualizado de visitas para diminuir o problema da separação da família, melhorar a qualidade da assistência e reduzir a ansiedade dos pacientes ${ }^{11-13}$. O fato de não ter controle sobre si leva à perda da independência do paciente o que traz angústia e sensação de incapacidade.

Os tubos, incluindo sondas, cateteres e cânulas, são invasivos, desconfortáveis e limitam a comunicação e a alimentação do paciente, aumentando o estresse na UTI juntamente com as demais privações que eles já experimentam neste ambiente ${ }^{3}$. A razão para o desconforto relacionado com o tubo traqueal possivelmente é devido à sucção endotraqueal, a qual os pacientes são regularmente submetidos para manter a permeabilidade da via aérea ${ }^{8}$. Este agressivo estímulo mecânico pode explicar porque os tubos são freqüentemente lembrados como importante causa de desconforto durante a internação em UTI ${ }^{14}$. O uso de sondas enterais também pode estar associado a desconforto e estresse em pacientes críticos, relacionados tanto ao seu incômodo mecânico, principalmente na sua introdução, quanto à privação do ato de comer. Como estes dispositivos são fundamentais para o seu manuseio na UTI, já foi proposto na unidade em questão, a utilização de um check-list para reavaliações freqüentes da necessidade de permanência dos tubos, a fim de que estes sejam retirados o mais precocemente possível.

Não dormir e ter dor causam desconforto físico e psicológico no paciente, o que somado às condições da internação aumentam seu estresse. Diversos estudos têm demonstrado que a privação do sono é um problema comum na UTI e está relacionado a alterações mentais nesses pacientes ${ }^{15-18}$. A privação do sono em UTI parece ter várias causas, incluindo a doença crônica do paciente, a presença de uma doença aguda sobreposta ou realização de procedimento cirúrgico, as medicações utilizadas e o próprio ambiente da UTI ${ }^{19,20}$. Medidas terapêuticas para melhorar esta condição devem ser direcionadas a todas as potenciais causas, com ênfase na criação de um ambiente favorável que facilite o início do sono, diminua o número de interrupções e possibilite a manutenção do ciclo sono-vigília nesses pacientes ${ }^{20}$.

O barulho dos alarmes parece preocupar mais os profissionais do que os próprios pacientes. A constan- 
te preocupação com alarmes faz parte da rotina dos profissionais de UTI, por estes se sentirem responsáveis pelas queixas físicas e psicológicas dos pacientes decorrentes do excesso de ruídos ${ }^{4}$. Um ambiente ruidoso dificulta um descanso confortável causando nos pacientes perturbações do sono, alterações psicológicas com desorientação e até mesmo ansiedade nas enfermeiras mais susceptíveis ${ }^{17}$. Os níveis de ruído hospitalar encontram-se excessivamente elevados, especialmente no ambiente de UTI, em decorrência dos inúmeros alarmes e equipamentos, além da conversação da própria equipe hospitalar. Diante disso, esse ambiente, que deveria ser silencioso e tranqüilo, torna-se ruidoso, transformando-se em grande fator de estresse e podendo gerar distúrbios fisiológicos e psicológicos tanto nos pacientes como nos funcionários desta unidade ${ }^{17}$.

Exposição a um ambiente com grande sobrecarga de estímulos sensitivos, dolorosos, ruído, aspiração traqueal e privação do sono pode exigir o uso de fármacos para controlar a ansiedade e inquietude dos pacientes e facilitar o sono. $\mathrm{O}$ adequado manuseio da sedação e analgesia na UTI também devem ser objetivados, visto que dor e ansiedade são estressores importantes neste meio. É fundamental assegurar analgesia suficiente aos pacientes e um dos aspectos mais importantes, nestes casos, é a determinação individual da dor. A intensidade, à qual a dor parece insuportável, varia não somente de paciente para paciente, mas também de acordo com o grau de ansiedade e com a sua cultura ${ }^{21}$.

Atualmente tem-se dado muita ênfase na necessidade de humanizar o ambiente da UTI. Um programa de humanização hospitalar supõe estabelecer um ambiente e cuidado humano e uma cultura de respeito e valorização não da doença, mas do ser humano que adoece, contemplando uma relação sujeito-sujeito e não sujeito-objeto ${ }^{22}$. A humanização das UTI está intimamente vinculada à atuação dos profissionais de saúde frente aos fatores estressantes ${ }^{4}$. É importante na atenção ao paciente o controle da dor e ansiedade, explicações sobre sua doença e tratamento em linguagem acessível, melhora da qualidade do sono, maior movimentação no leito, política de visitas aberta, respeito à privacidade, conforto e apoio psicológico e emocional ${ }^{7}$. O envolvimento com o paciente e a família é um pré-requisito essencial para humanizar. Porém, este aspecto deveria ser trabalhado e discutido com a equipe para não gerar angústia ou sentimento de impotência, levando, com isso, à negação e ao distanciamento como mecanismos de defesa ${ }^{23}$. Na unidade avaliada a equi- pe de psicologia desempenha um papel fundamental neste contexto, aproximando a família do ambiente da UTI e promovendo melhor interação entre esta, o paciente e a equipe de saúde.

Os resultados do presente estudo devem ser considerados ainda no contexto de algumas limitações relacionadas principalmente aos critérios de inclusão dos pacientes, visto que todos se encontravam conscientes e em ventilação espontânea. Esta amostra não retrata necessariamente o perfil dos pacientes internados em UTI, que, na maioria das vezes, encontram-se inconscientes, desorientados, em ventilação mecânica e com algum grau de sedação. Desta forma, outros trabalhos que avaliam os pacientes após a alta da UTI ou após um período maior de internação, poderiam contribuir para um melhor estudo dos estressores nesta população. No entanto, é comum que os pacientes que foram sedados durante a internação na UTI tenham utilizado fármacos com potencial amnésico e não tenham recordações desse período ${ }^{24}$. Granja e col. avaliaram 464 pacientes de 10 UTI em Portugal, seis meses após a alta, e encontraram que $20 \%$ a $55 \%$ deles apresentavam amnésia sobre todo o período em que estiveram internados na UTI ${ }^{8}$.

\section{CONCLUSÕES}

Através deste trabalho, pode-se contribuir para a avaliação dos principais estressores para pacientes internados em UTI. Pouco contato com a família, presença de tubos e não ter controle do corpo foram os fatores mais freqüentes entre os pacientes. Tanto os familiares como os profissionais de saúde apresentaram escores totais de estresse superiores aos encontrados nos pacientes, superestimando o estresse quando comparado aos próprios pacientes e às vezes divergindo quanto aos principais estressores. A identificação destes estressores é importante para a implementação de medidas que, atuando nesses fatores, possam facilitar a humanização do ambiente da UTI. Trabalhos posteriores poderão avaliar melhor o efeito dessas medidas na qualidade da assistência prestada aos pacientes internados na UTI e no seu prognóstico.

\section{REFERÊNCIAS}

01. Gois CFL, Dantas RAS - Estressores em uma unidade pós-operatória de cirurgia torácica: avaliação da enfermagem. Rev Latino-Am Enfermagem, 2004;12:22-27.

02. Hewitt $\mathrm{J}$ - Psycho-affective disorder in intensive care units: a review. $J$ Clin Nurs, 2002;11:575-584.

03. Novaes MA, Aronovich A, Ferraz MB et al - Stressors in ICU: patients' 


\section{ANÁLISE DE ESTRESSORES PARA O PACIENTE EM UNIDADE DE TERAPIA INTENSIVA}

evaluation. Intensive Care Med, 1997;23:1282-1285.

04. Novaes MA, Knobel E, Bork AM et al - Stressors in ICU: perception of the patient, relatives and health care team. Intensive Care Med, 1999;25:1421-1426.

05. Ballard KS - Identification of environmental stressors for patients in a surgical intensive care unit. Issues Ment Health Nurs, 1981;3:89-108.

06. Nastasy EL - Identifying environmental stressors for cardiac surgery patients in SICU. In: Proceedings of the $12^{\text {th }}$ Annual National Teaching Institute of AACN (1985). Newport Beach, Calif.:AACN 357.

07. Gomes AMCG, Santos PAJ - Humanização em Medicina Intensiva, em: Amaral JLG - Clínicas Brasileiras de Medicina Intensiva: Sedação, Analgesia e Bloqueio Neuromuscular em UTI. São Paulo, Atheneu, 1997;1346.

08. Granja C, Lopes A, Moreira S et al - Patients' recollections of experiences in the intensive care unit may affect their quality of life. Crit Care, 2005;9:R96-R109.

09. Cornock MA - Stress and the intensive care patient: perceptions of the patients and nurses. J Adv Nurs, 1998;27:518-527.

10. Cochran J, Ganong LH - A comparison of nurses' and patients' perceptions of intensive care unit stressors. J Adv Nurs, 1989;14:1038-1043.

11. Correa AK, Sales, CA, Soares $L$ - A família do paciente internado em terapia intensiva: concepções do enfermeiro. Acta Sci, 2002;24:811-818.

12. Souza RMC - Visitas em UTI: subsídios para reflexão. Rev Paul Hospitais, 1988;36:24-29.

13. Azoulay E, Pochard F, Chevret $S$ et al - Family participation in care to the critically ill: opinions of families and staff. Intensive Care Med, 2003;29:1498-1504.

14. Van de Leur JP, Van der Schans CP, Loef BG et al - Discomfort and fac- tual recollection in intensive care unit patients. Crit Care, 2004;8:R467R473.

15. Pimentel-Souza F, Carvalho JC, Siqueira AL - Noise and the quality of sleep in two hospitals in the city of Belo Horizonte, Brazil. Braz J Med Biol Res, 1996;29:515-520.

16. Hodge B, Thompson JF - Noise pollution in the operating theatre. Lancet, 1990;355:891-894.

17. Pereira RP, Toledo RN, Amaral JLG et al - Qualificação e quantificação da exposição sonora ambiental em uma unidade de terapia intensiva. Rev Bras Otorrinolaringol, 2003;69:766-771.

18. Freedman NS, Kotzer N, Schwab RJ - Patient perception of sleep quality and etiology of sleep disruption in the intensive care unit. Am J Respir Crit Care Med, 1999;159:1155-1162.

19. Weinhouse GL, Schwab RJ - Sleep in the critically ill patient. Sleep, 2006;29:707-716.

20. Krachman SL, D'Alonzo GE, Criner GJ - Sleep in the intensive care unit. Chest, 1995;107:1713-1720

21. Slullitel A, Sousa AM - Analgesia, sedação, bloqueio neuromuscular em UTI. Medicina (Ribeirão Preto), 1998;31:507-516.

22. Backes DS, Lunardi-Filho WD, lunardi VL - The humanization process of the hospital environment centered around the worker. Rev Esc Enferm USP, 2006;40:221-227.

23. Vila VSC, Rossi LA - O significado cultural do cuidado humanizado em unidade de terapia intensiva: "muito falado e pouco vivido". Rev LatinoAm Enfermagem, 2002;10:137-144.

24. Samuelson K, Lundberg D, Fridlund B - Memory in relation to depth of sedation in adult mechanically ventilated intensive care patients. Intensive Care Med, 2006;32:660-667. 